\title{
Development of a Real-time Bionic Voice Generation System based on Statistical Excitation Prediction
}

\author{
Farzaneh Ahmadi \\ Nagoya University \\ Nagoya/Japan \\ farzaneh.ahmadi@g.sp.m.is.nag \\ oya-u.ac.jp
}

\author{
Kazuhiro Kobayashi \\ Nagoya University \\ Nagoya/Japan \\ kobayashi.kazuhiro@g.sp.m.is.n \\ agoya-u.ac.jp
}

\author{
Tomoki Toda \\ Nagoya University \\ Nagoya/Japan \\ tomoki@icts.nagoya-u.ac.jp
}

\begin{abstract}
Despite the emergent progress in many fields of bionics, larynx amputees still lack a functional Bionic Voice source to overcome their voice disability. We have established the Pneumatic Bionic Voice (PBV) as a promising technology to generate a voice for larynx amputees. This summary provides an overview of our efforts to implement the PBV system in real-time together with a demonstration of its results.

The Pneumatic Bionic Voice prosthesis is an electronic adaptation of an old school mechanical voice source called the Pneumatic Artificial Larynx (PAL). The PAL is a noninvasive voice prosthesis, with an exceptionally high-quality voice, driven exclusively by respiration. This work proposes an approach to statistically model the PAL's voice generation mechanism from the respiration and to implement it in a real-time PBV system that larynx amputees can use to generate voice in continuous speech.
\end{abstract}

\section{Author Keywords}

Pneumatic Bionic Voice; Voice Prosthesis; Pneumatic Artificial Larynx; PAL; Voice conversion; Laryngectomy.

\section{CSS Concepts}

- Human-centered computing Accessibility; Accessibility Technologies;

\section{INTRODUCTION}

Larynx amputees (laryngectomees) lose their larynx in advanced stages of throat cancer. Losing the larynx leads to the loss of vocal folds and the voice as the excitation source of speech. Voice prostheses aim to regenerate this excitation source. Similar to the missing vocal folds, larynx amputees shape this artificial excitation source into speech by moving their face and lips muscles. Among many voice prostheses, the Pneumatic Artificial Larynx still stands out, as the only non-invasive voice prosthesis with an exceptionally high voice quality [1-5]. It is interesting that the PAL is driven

Permission to make digital or hard copies of part or all of this work for personal or classroom use is granted without fee provided that copies are not made or distributed for profit or commercial advantage and that copies bear this notice and the full citation on the first page. Copyrights for third-party components of this work must be honored. For all other uses, contact the Owner/Author:

Farzaneh Ahmadi farzaneh.ahmadi@g.sp.m.is.nagoya-u.ac.jp

ASSETS '19, October 28-30, 2019, Pittsburgh, PA, USA

(C)2019 Copyright is held by the owner/author(s).

Publication rights licensed to:

ACM ISBN 978-1-4503-6676-2/19/10.

https://doi.org/10.1145/3308561.3354591 exclusively by respiration to generate such high-quality voice. This has encouraged us to estimate the voice generation function of the PAL and employ it in a Pneumatic Bionic Voice source as its electronic adaptation [6].

The design approach of a PBV is to mimic the functionality of the PAL both in terms of controlling voice generation and reaching a similar degree of naturalness. We have already captured the voice onset/offset control of the PAL with $98.4 \%$ accuracy. This enabled us to develop the first realtime Bionic Voice source which a laryngectomee used to control using his respiration to speak in slow/fast speech rates [6]. To improve the PBV's naturalness, we developed a statistical respiration to voice conversion system to generate a voice close to the PAL source waveform from respiration. Using this framework in our design, substantially improved the Bionic Voice quality in our offline simulations and was verified through objective and subjective listening tests [7].

This paper takes the next step and develops this statistical Pneumatic Bionic Voice source prediction framework in real-time. We are excited to demonstrate this system in ASSETS 2019 and to proceed with its upcoming trials with the laryngectomees in the near future.

\section{REAL-TIME PBV SOURCE GENERATION SYSTEM}

\section{System Overview}

When a laryngectomee loses their larynx, an opening in the neck (stoma) is generated for them to breathe. The PAL is placed externally between this stoma and the mouth and similar to a whistle is driven by the variations of the pressure at its two ends, $p_{m}(t), p_{s}(t)$ (Figure 1). In that sense, the PAL acts as a mechanical model of the human larynx with a fixed pair of vocal folds, driven exclusively by respiration to generate the excitation source waveform of speech: $\mathrm{e}(t)$.
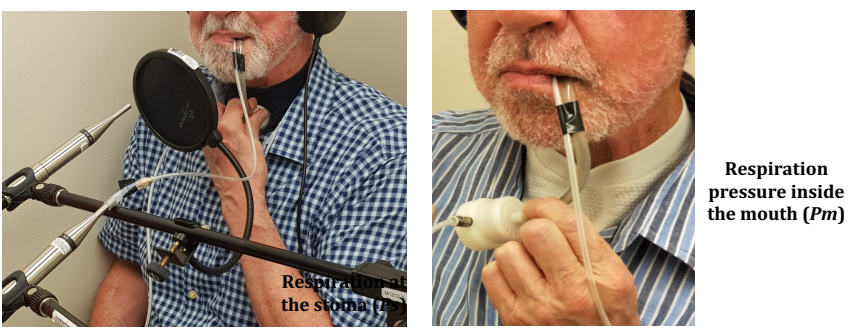

Figure 1. Respiration drive of the PAL source as a laryngectomee participant uses the PAL to speak with. 
To reach a similar high quality, the PBV system has to estimate, $\mathrm{e}(t)$, the source excitation waveform of the PAL, from the underlying respiration signals. For this, we adopt the general statistical voice conversion framework by Toda et al. [8] and modify it to a respiration to voice conversion system [6]. The PAL's driving pressure $p_{m}(t), p_{s}(t)$, are the inputs and the PAL's generated voice waveform, $\mathrm{e}(t)$, is the intended output of this conversion system.

\section{Statistical Source Excitation Prediction}

Figure 2 summarizes the structure of the real-time PBV system. To estimate the source, $\mathrm{e}(t)$ is broken into three components of: spectral envelope, $f_{0}$ and aperiodicity, extracted with WORLD vocoder [9]. The spectral envelope is further parameterized into 24 mel-cepstral coefficients. A set of 14 features are extracted from the respiration signal [10]. In the training phase, three Gaussian Mixture Models (GMMs) are trained for mel-cepstrum, aperiodicity and $f_{0}$ component estimation using joint probability density of respiration/voice features trajectory. The trained GMMs are used in the test phase and maximum likelihood parameter generation (MLPG) [11] of a trajectory of estimated values is used to achieve the final $f_{0}$, mel-cepstrum and the aperiodicity values for each frame. The estimated PAL source waveform $(\hat{e}(t))$ is then synthesized by combining the three estimated components using a vocoder [9].

The real-time PBV system follows the same concept as the offline PBV [7] except for one main difference. In the offline PBV system, a batch-type version of the maximum likelihood parameter generation (MLPG) is used [11]. So, in the conversion stage of the system, the spectral parameters of multiple frames are generated simultaneously to benefit from feature correlations between frames. This is not feasible in the real-time system where a frame-by-frame low-delay MLPG should be employed [12]. This low-delay MLPG approach uses a recursive parameter generation algorithm already proven feasible in speech synthesis [13].

\section{Implementation Details}

The real-time PBV system receives its respiration input signals $\left(p_{m}(t), p_{s}(t)\right)$ at $1 \mathrm{kHz}$ through a UDP port of an Arduino $^{\mathrm{TM}}$ based pressure sensing system [6]. The UDP (also used in VoIP applications) is used due to its connectionless nature to eliminate any waiting time for the respiration signal to arrive. The respiration input and voice output of the system are windowed at overlapping $10 \mathrm{~ms}$ and $25 \mathrm{~ms}$ frames respectively, both with $5 m s$ of shift. Every $5 m s$, the PBV system receives one frame $(10 \mathrm{~ms})$ of respiration and generates $5 \mathrm{~ms}$ of audio (voice) and sends it to the sound card.

The feature sequence in the real-time system is shaped similar to the offline PBV system with the analysis frame in the middle ( $n$ frames ahead and $n$ frames before the analysis frame). This leads to $n$ frames of look-ahead delay ( $n * 5 m s$ of frame-shift) in the real-time PBV system (in addition to hardware delays). With $n=2$ frames in this implementation the real-time estimation will have a delay of more than $10 \mathrm{~ms}$.

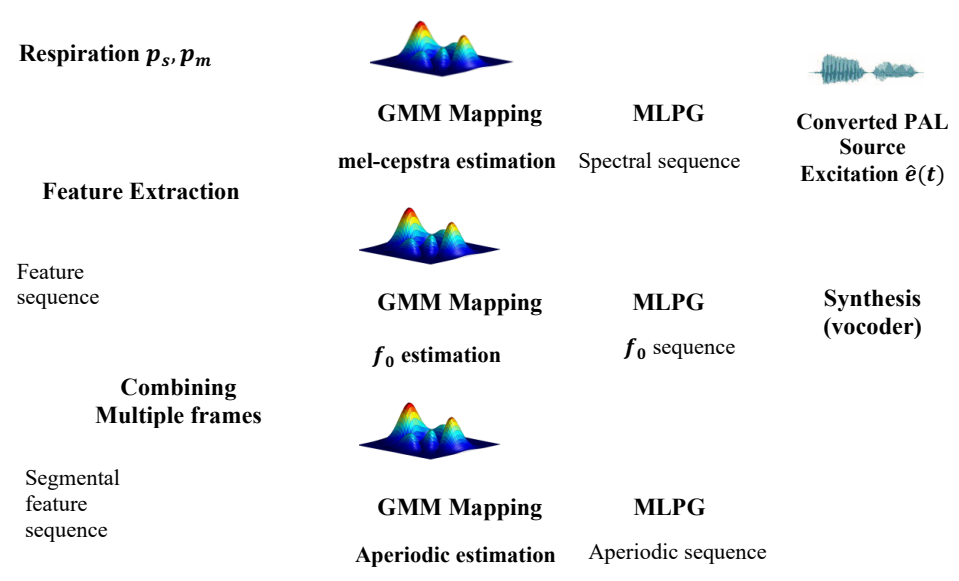

Figure 2. Real-time respiration to voice conversion system.

The system was implemented in $\mathrm{C}++$ on an iMac computer with $32 \mathrm{~GB}$ of RAM and a $4.2 \mathrm{GHz}$ Intel Core $\mathrm{i} 7$ processor. To maintain a real-time performance, the system was synchronized with an independent external clock. For this, the system was implemented using two threads: a) one thread to perform the respiration to voice conversion, i.e. reading each respiration frame, estimating the voice and placing the synthesized audio frame in a certain memory address, b) an independent audio callback thread run by the OS to take the synthesized frame from that memory address and operate the sound card to play the synthesized sound frame.

\section{EVALUATION}

The average time delay between each frame of respiration input and the synthesized audio output for that frame was measured to be $23 \mathrm{~ms}$ (sum of look-ahead and hardware delays). This led to a straightforward real-time performance in synthesizing a glitch-free audio by the soundcard.

The objective comparison of the real-time and offline systems showed a close match. The sum of the difference between the respiration feature extraction methods of real-time and offline PBV systems for respiration recordings of 45 seconds was in the order of $10^{-7}$. The estimation accuracy of $f_{0}$ was $98 \%$ in real-time compared to the offline PBV and the two system's Mel-Cepstral distortion of the estimated spectrum coefficients was $3.9 \mathrm{~dB}$. To evaluate the system as a whole, the estimated PAL excitation source waveforms were used to re-synthesize a copy of their corresponding PAL speech signal in an approach explained in our previous work [6]. The simulated speech quality of the offline and real-time PBV systems will be provided as part of the demonstration.

\section{CONCLUSION}

We implemented the first real-time Pneumatic Bionic Voice source prediction system and will demonstrate its performance in comparison with the reference PAL device. Our next step is to proceed with live trials of this system with larynx amputees.

\section{ACKNOWLEDGMENTS}

The authors wish to thank Japan's Society for Promotion of Science to support part of this work through an Invitational Research Fellowship, 2019. This work was partly supported by JST, PRESTO Grant Number JPMJPR1657. 


\section{REFERENCES}

[1] B. Weinberg, A. Riekena. 1973. Speech produced with the Tokyo artificial larynx, J Speech Hear Disord, 38, 3:383-389.

[2] R. Goode. 1975. Artificial laryngeal devices in postlaryngectomy rehabilitation, The Laryngoscope, 85, 4: 677-689.

[3] J. Xu, X. Chen, M. Lu, M. Qiao. 2009. Perceptual evaluation and acoustic analysis of pneumatic artificial larynx," Otolaryng Head Neck, 141, 6:776-780.

[4] M. Ng, C. Kwok, S. Chow. 1997. Speech performance of adult Cantonese-speaking laryngectomees using different types of alaryngeal phonation, $J$ Voice, 11, 3: 338-344.

[5] S. Bennett, B. Weinberg. 1973. Acceptability ratings of normal, esophageal, and artificial larynx speech, J Speech, Lang Hear Res, 16,4: 608.

[6] F. Ahmadi, F. Noorian, D. Novakovic, A. van Schaik. 2018. A pneumatic Bionic Voice prosthesisPre-clinical trials of controlling the voice onset and offset," PloS one, 13, 2:e0192257.

[7] F. Ahmadi, T. Toda. 2018. Designing a Pneumatic Bionic Voice Prosthesis-A Statistical Approach for Source Excitation Generation, in Interspeech 2018, 3142:3146.

[8] T. Toda, M. Nakagiri, K. Shikano. 2012. Statistical voice conversion techniques for body-conducted unvoiced speech enhancement, IEEE Trans Audio, Speech, 20, 9:2505-2517.
[9] M. Morise, F. Yokomori, K. Ozawa. 2016. WORLD: a vocoder-based high-quality speech synthesis system for real-time applications, IEICE Trans Inf Systems, 99, 7: 1877-1884,

[10] F. Ahmadi, M. A. Ribeiro, M. Halaki. 2014. Surface electromyography of neck strap muscles for estimating the intended pitch of a bionic voice source, In IEEE Biomedical Circuits and Systems Conference (BioCAS), 37-40.

[11] K. Tokuda, T. Yoshimura, T. Masuko, T. Kobayashi, T. Kitamura. 2000. Speech parameter generation algorithms for HMM-based speech synthesis, In IEEE International Conference on Acoustics, Speech, and Signal Processing, 3: 1315-1318.

[12] T. Toda, T. Muramatsu, H. Banno. 2012. Implementation of computationally efficient real-time voice conversion, In Thirteenth Annual Conference of the International Speech Communication Association.

[13] K. Tokuda, T. Kobayashi, S. Imai. 1995. Speech parameter generation from HMM using dynamic features, In International Conference on Acoustics, Speech, and Signal Processing, 1:660-663. 